\title{
A superfine structure in solar microwave bursts
}

\author{
G. P. Chernov ${ }^{1,2, \star}$, Y. H. Yan ${ }^{1}$, and Q. J. Fu ${ }^{1}$ \\ 1 National Astronomical Observatory, Chinese Academy of Science, 20A, Datun Road, Beijing 100012, PR China \\ e-mail: yyh@bao.ac.cn \\ 2 Izmiran, Troitsk, Moscow region 142190, Russia \\ e-mail: gchernov@izmiran.troitsk.ru
}

Received 27 January 2003 / Accepted 25 February 2003

\begin{abstract}
We have observed in the microwave range (with the radio spectrometer of the Huairu station (Beijing, NAOC) around $3 \mathrm{GHz}$ ) the fine structure of solar radio bursts called zebra patterns and fiber bursts (seen drifting on the frequency stripes in emission and in absorption on the background burst continuum emission). In all seven observed bursts we discovered a new effect: zebra stripes have a superfine structure, consisting of numerous fast spikes with duration at a limit of the time resolution of the spectrometer, $8 \mathrm{~ms}$. Since for zebra patterns and microwave spikes different emission mechanisms were proposed, these new observations require us to revise known theories. An alternative model of microwave millisecond spikes is based on the coupling of plasma waves $(l)$ with ion-sound waves $(s): l+s \rightarrow t$. Since the main features of zebra stripes and fiber bursts are similar, we consider the the zebra pattern of these bursts as whistler manifestations. Whistlers $(w)$ yield a principal contribution in the fine structure radio emission $(t)$ by coupling with Langmuir waves at sum as well as difference frequencies: $\omega_{l} \pm \omega_{w}=\omega_{t}$. Allowance for the conversion of ion-sound waves into whistlers (and inversely in a pulsating regime) enables us to identify the zebra pattern consisting of spikes as a simultaneous manifestation of both those processes $(l+s \rightarrow t$ and $l+w \rightarrow t)$ in radio sources, related to magnetic reconnection above flare regions.
\end{abstract}

Key words. Sun: flares - Sun: radio radiation

\section{Introduction}

A superfine structure was observed as a part of zebra pattern around $3 \mathrm{GHz}$ : each zebra stripe consisted of millisecond spikes. Stripes in emission and absorption called zebra pattern structure (ZPS) and fiber-bursts (FB) (or bursts with intermediate frequency drift) are well-known fine structures in the meter and decimeter continuum emission of type IV solar radio bursts (Slottje 1981). Since the end of the seventies, microwave observations of solar emission with high temporal and spectral resolution have also shown fine structures: millisecond spikes (with the duration of single spikes of a few tens of milliseconds), and fast pulsations, usually superimposed on a smooth continuum emission (Benz 1993). We could also find some indications of a probable zebra pattern in the microwave range in Isliker \& Benz (1994).

High time and frequency resolution data have improved our studies of similar fine structures in star flares. New microwave spectrometers of NAOC (China, Huairou station) with higher resolution $(10 \mathrm{MHz}$ and $8 \mathrm{~ms}$ ) can observe detailed zebra patterns and fiber bursts at high frequencies, $2.6-7.6 \mathrm{GHz}$ (Chernov et al. 2001b). In the microwave range the same

Send offprint requests to: G. P. Chernov, e-mail: gchernov@izmiran.troitsk.ru

* Constant address: IZMIRAN, Troitsk, Moscow region 142190, Russia. variety of fine structure is observed. Thus the relative frequency parameters of zebra structures in microwave and meter wave bands appear identical. This testifies to the identical nature of the formation of fine structure in different frequency ranges.

Some recent observations with a time resolution of $0.1 \mathrm{~s}$, using Ondřejov radiospectrographs in the range $0.8-4 \mathrm{GHz}$, have allowed us to find new properties of zebra structures and to verify theoretical models (Ledenev et al. 2001; Karlicky et al. 2001; Sawant et al. 2002).

Concerning the interpretation of such fine structures, the interaction of plasma electrostatic waves $(l)$ with whistler waves $(w)$ (generated by the same fast particles with loss-cone anisotropy) is a well-accepted emission mechanism for fiber bursts: $l+w \rightarrow t$ with freely escaping electromagnetic waves $(t)$ in the ordinary (O) mode (Kuijpers 1975). Zebra-patterns are more complicated structures, and many mechanisms have been proposed to explain them (Kuijpers 1975; Zheleznykov \& Zlotnik 1975; Chernov 1976; Mollwo 1983, 1988; Winglee \& Dulk 1986; Ledenev et al. 2001; Karlicky et al. 2001). The majority are based on electrostatic emission at double plasma resonance:

$\omega_{\mathrm{UH}}=\left(\omega_{\mathrm{Pe}}^{2}+\omega_{\mathrm{Be}}^{2}\right)^{1 / 2}=s \omega_{\mathrm{Be}}$

with $\omega_{\mathrm{UH}}-$ upper hybrid frequency, $\omega_{\mathrm{Pe}}-$ electron plasma frequency, $\omega_{\mathrm{Be}}-$ electron cyclotron frequency and $s-$ integer harmonic number. The most advanced model in this 
category, proposed by Wenglee \& Dulk (1986), is based on cyclotron non-saturated maser emission of electrostatic waves by a loss-cone electron distribution. However some difficulties remain with all these versions: the frequency separation between zebra stripes $\Delta f_{\mathrm{e}}$ corresponds to approximately the cyclotron frequency and this is hard to reconcile with its often irregular variation with frequency; the magnetic field deduced from $\Delta f_{\mathrm{e}}$ seems too low for the flare region and we are faced with the difficulty of having plasma $\beta \approx\left(v_{s} / v_{A}\right)^{2} \ll 1$ (a well accepted value for magnetic traps in active regions).

Ledenev et al. (2001) have chosen a scale of heights of a magnetic field much less than the scale of heights of plasma density. They have assumed that radiation originates in the second harmonic $f_{\mathrm{UH}}$ and they have determined higher values of magnetic field strength $B$ (comparable with values from Chernov et al. 2001b in models with whistlers). However, ZPS are usually strongly polarized and very seldom have moderate polarization, suggesting radiation in the first harmonic. Thus values $(B)$ appear overestimated by more than twofold as the number of harmonics $s$ is arbitrarily chosen. In Sawant et al. (2002) for the first time the fragment of ZPS on harmonic frequencies (1700 and $3400 \mathrm{MHz}$ ) is discussed; such a trend was predicted in the model of Ledenev et al. (2001). However in the absence of information on polarization and also in the absence of any conformity of zebra-stripes at harmonic frequencies, a certain doubt remains about the reality of harmonic structure. An important point missing in previous theories is that a losscone distribution generates whistlers, which in turn affect the electron velocity distribution (Chernov 1996).

Many features of zebra stripes and fiber bursts are similar: both structures represent parallel drifting stripes in emission and in absorption. This led Chernov (1976, 1989) to propose a common interpretation for these two kinds of fine structure based on the coupling of plasma wave and whistlers, but in different conditions of the whistler instability: for fiber bursts at the cyclotron resonance with a normal Doppler effect when whistlers propagate along the magnetic loops; for zebra-stripes, the anomalous Doppler effect, when whistlers propagate at different angles to magnetic force lines (Maltseva $\&$ Chernov 1989; Chernov et al. 1998). A system of quasistanding whistler packets is driven by a loss-cone distribution of fast electrons in the entire magnetic trap at the cyclotron resonance:

$\omega_{w}-k_{\|} v_{\|}-s \omega_{\mathrm{Be}}=0$

with $s=+1$ (normal Doppler effect) or $s=-1$ (anomalous Doppler effect) depending on the form of the distribution function (Maltseva \& Chernov 1989), $k_{\|}, v_{\|}$being the whistler wave number and fast electron velocity, both parallel to the magnetic field, $\omega_{w}$ - whistler wave frequency.

The theory of electron cyclotron maser instability (ECMI) seemed to be the most attractive model to account for the main properties of microwave spikes (see the exhaustive review by Fleishman \& Melnikov 1998 and Chernov et al. 2001a and references therein). The main problems with ECMI are the escape of the radiation through the outer corona without absorption at the third cyclotron level and the emission of only $\mathrm{X}$ and $\mathrm{Z}$ magnetoionic modes in a region where the ratio of electron plasma and cyclotron frequencies $\omega_{\mathrm{Pe}} / \omega_{\mathrm{Be}} \leq 0.5$, meaning a too strong magnetic field. Source dimensions of microwave spikes are usually proposed to be $\leq 10^{8} \mathrm{~cm}$ and brightness temperatures $T_{\mathrm{b}} \geq 10^{13} \mathrm{~K}$ (Fleishman \& Melnikov 1998).

In the decimeter range, spikes were sometimes observed in the events with zebra structure (Kuijpers et al. 1981) which allowed Barta \& Karlicky (2001) to connect the radiation of dmspikes with the mechanism of double plasma resonance, but in conditions of turbulent plasma near points of the magnetic reconnection. Thus radiation of a single spike can develop from different narrow magnetic loops that can explain the unexpectedly large sizes of their sources comparable to the size of the whole flare region (Altyntsev et al. 1998). Conditions of turbulent plasma with strongly irregular gradients of density and magnetic field are postulated in Barta \& Karlicky (2001).

In this paper we present observations of a new property of ZPS. We describe and analyze seven bursts with ZPS and FB and in all 7 observed bursts we discovered a completely new effect: zebra stripes have their own superfine structure, consisting of numerous fast spikes with duration at the limit of the time resolution of the spectrometer, $\leq 8 \mathrm{~ms}$. We consider the fine structure of these bursts as whistler manifestations. Whistlers $(w)$ yield a principal contribution in the fine structure radio emission $(t)$ by coupling with Langmuir waves $(l)$ at sum as well as difference frequencies (Chernov 1976): $\omega_{l} \pm \omega_{w}=\omega_{t}$.

Taking into account our new observations of zebra stripes consisting of millisecond spikes, it should be noted that any version of the emission mechanism of zebra patterns proposes an inverse ratio $\omega_{\mathrm{Pe}} / \omega_{\mathrm{Be}} \gg 1$. Altyntsev et al. (1998) explain their observations of spikes by a plasma emission mechanism. To avoid such difficulties, an alternative model of microwave millisecond spikes was proposed in Chernov et al. (2000a), based on the coupling of plasma waves with ion-sound waves $(s): l+s \rightarrow t$. Allowance for the conversion of ion-sound $(s)$ waves into whistlers (and inversely) (Chernov 1989) enables us to identify the zebra-pattern consisting of spikes as simultaneous manifestations of both those processes $(l+s \rightarrow t$ and $l+w \rightarrow t$ ) in radio sources, related to magnetic reconnection above flare regions.

\section{New observations}

There were seven events with ZPS and spikes observed with the broadband radio spectrometer at Huairu station (Beijing, NAOC) (Fu et al. 1995) in the frequency range 2.6-3.8 GHz. The characteristics of these bursts are listed in Table 1, and examples from three events are shown in Figs. 1-3. The wealth of fine structure does not always correspond to the size of the chromospheric flares and the intensity of the radio bursts. The relatively weak event of April 15, 1998 was very rich in spikes and ZPS. The event on October 29, 2000 was the most rich in fine structures (see Chernov et al. 2001b). Zebra patterns were usually observed after the burst maximum in all events. The most important parameter of ZPS in any model is magnetoionic wave mode $(\mathrm{O}, \mathrm{X})$ and to define the magnetic polarity in the radio source, all accessible data were used, in particular flare images in extreme ultraviolet lines (cosmic data from SOHO/EIT, 
Table 1. Parameters of seven radio bursts with ZPS and millisecond spikes.

\begin{tabular}{|c|c|c|c|c|c|c|c|c|c|c|c|}
\hline $\begin{array}{l}\text { Date } \\
\text { time UT }\end{array}$ & $\begin{array}{c}d \\
(\min )\end{array}$ & $\begin{array}{l}\text { Flare, } \\
\text { coord. }\end{array}$ & $\begin{array}{c}\text { AR } \\
\text { NOAA }\end{array}$ & $\begin{array}{l}\text { Flux } \\
\text { (sfu) }\end{array}$ & $\begin{array}{c}\Delta f \\
\mathrm{GHz}\end{array}$ & $\begin{array}{c}\Delta f_{\mathrm{e}} \\
\mathrm{MHz}\end{array}$ & $\begin{array}{c}p \\
\mathrm{R}, \mathrm{L}\end{array}$ & $\begin{array}{c}m \\
\mathrm{o}, \mathrm{x}\end{array}$ & $\begin{array}{c}M \\
\mathrm{~N}, \mathrm{~S}\end{array}$ & $N$ & $n$ \\
\hline $\begin{array}{l}15.04 .98 \\
07: 59\end{array}$ & 3 & $\begin{array}{c}\text { SN,C8.8 } \\
\text { N29W15 }\end{array}$ & 8203 & 300 & $\begin{array}{c}3.45- \\
3.8\end{array}$ & $65-70$ & $\mathrm{R}$ & 0 & $\mathrm{~S}$ & 2 & 3 \\
\hline $\begin{array}{l}21.01 .00 \\
05: 07\end{array}$ & 1 & * C6.7 & 8837 & 140 & $\begin{array}{c}2.6- \\
3.3\end{array}$ & 75-100 & $\mathrm{L}$ & o & $\mathrm{N}$ & 2 & 5 \\
\hline $\begin{array}{l}29.10 .00 \\
02: 19\end{array}$ & 16 & $\begin{array}{l}2 \mathrm{~B}, \mathrm{C} 4.4 \\
\mathrm{~S} 25 \mathrm{E} 35\end{array}$ & 9209 & 1800 & $\begin{array}{c}2.6- \\
3.1\end{array}$ & $65-80$ & $\mathrm{R}$ & o & $S$ & 14 & $2-6$ \\
\hline $\begin{array}{l}24.11 .00 \\
05: 00\end{array}$ & 2 & $\begin{array}{l}3 \mathrm{~B}, \mathrm{X} 2.0 \\
\text { N20W05 }\end{array}$ & 9236 & 2200 & $\begin{array}{c}2.6- \\
3.8\end{array}$ & $55-60$ & $\begin{array}{l}\mathrm{L} \\
\mathrm{R}\end{array}$ & o & $\mathrm{N}$ & 6 & $1-3$ \\
\hline $\begin{array}{l}25.11 .00 \\
01: 05\end{array}$ & 4 & $\begin{array}{c}\text { 2N,M8.2 } \\
\text { N07E50 }\end{array}$ & 9240 & 11000 & $\begin{array}{c}2.6- \\
3.8\end{array}$ & 50 & $\begin{array}{l}\mathrm{L} \\
\mathrm{R}\end{array}$ & o & $\mathrm{N}$ & 4 & $1-6$ \\
\hline $\begin{array}{l}10.04 .01 \\
05: 26\end{array}$ & 8 & $\begin{array}{l}3 B, X 2.3 \\
\text { S23W09 }\end{array}$ & 9415 & 4000 & $\begin{array}{c}2.6- \\
3.1\end{array}$ & 47 & $\begin{array}{l}\mathrm{Lz} \\
\mathrm{Rf}\end{array}$ & $\begin{array}{l}\mathrm{o} \\
\mathrm{o}\end{array}$ & $\begin{array}{l}\mathrm{N} \\
\mathrm{S}\end{array}$ & 3 & 3 \\
\hline $\begin{array}{l}19.10 .01 \\
00: 55\end{array}$ & 36 & $\begin{array}{c}\text { 2B,X1.6 } \\
\text { N16W18 }\end{array}$ & 9661 & 1400 & $\begin{array}{c}2.6- \\
3.0\end{array}$ & $55-75$ & $\mathrm{R}$ & o & S & 22 & $3-6$ \\
\hline
\end{tabular}

$d$ - Duration of fine structure; AR, NOAA - number of NOAA active region; Flux (sfu) - maximum of radio flux at $2695 \mathrm{MHz}$ in solar flux units; $\Delta f(\mathrm{GHz})$ - frequency range of ZPS and $\mathrm{FB} ; \Delta f_{\mathrm{e}}$ - frequency separation between emission stripes in ZPS; $p$ (R, L) - right, left sign of circular polarization (Lz for ZPS, Lf for FB); $m(\mathrm{o}, \mathrm{x})$ - magnetoionic wave mode (ordinary, extraordinary); $M(\mathrm{~N}, \mathrm{~S})-$ magnetic polarity nearest to the radio source; $N$ - number of cases (of duration usually of some seconds) with ZPS and FB in the event; $n$ - number of zebra stripes; *- no optical data.

TRACE), and in soft X-rays (Yohkoh/SXT), as well as radio source positions taken with the Siberian Solar radio Telescope (SSRT, 5.7 GHz) and Nobeyama Radioheliograph at $17 \mathrm{GHz}$.

The wave mode was defined with confidence in almost all events as the ordinary (O)-mode, even when in one event ZPS and FB had different signs of polarization, as e.g. in the event of April 10, 2001: ZPS had left-hand and FB had right-hand circular polarization. In this case the radio source of ZPS was located above the tail spot while FB was above the leading spot in active region 9415, according to source positions of SSRT at $5.7 \mathrm{GHz}$, and locations of new bright loops in some TRACE images in the EUV line $171 \AA$. During two bursts on November 24 and 25, 2000, zebra-stripes appeared in different polarization channels simultaneously, and in both cases radio sources had a double structure above areas of different magnetic polarity.

In the two top panels of Fig. 1 two spectra of the weak event of January 21, 2000 in left and right hand polarization are shown (the degree of polarization was moderate). A cloud of emission (with rounded borders) consists of spikes, as well as zebra stripes with negative and positive frequency drift. The emission bandwidth of zebra stripes is approximately equal to the bandwidth of spikes $(50-100 \mathrm{MHz})$, which are practically vertical white stripes with duration at the limit of the time resolution, $8 \mathrm{~ms}$. We could conclude that the source dimension of an isolated zebra stripe is about equal to the source dimension of spikes, and therefore their brightness temperatures are also about the same, $T_{\mathrm{b}} \geq 10^{13} \mathrm{~K}$.

A fragment of similar structure in the strong radio burst of November 25, 2000 is present in the bottom panel of Fig. 1. The spectrum is shown in one left polarization channel, because the emission was fully polarized at this moment. The emission on the whole includes numerous spikes, as well as zebra stripes showing oscillating frequency drift. The frequency separation between stripes and the bandwidth of each stripe are smaller than in the upper panels $(\sim 50 \mathrm{MHz})$.

The event of November 24, 2000 was the most spectacular regarding the superfine structure under discussion. Figure 2 shows an isolated zebra-stripe at the beginning of the burst evolving over six seconds into double and triple stripes, all consisting of spikes in left polarization. At the same time we see radio emission in right polarization without any spiky structure. The event was very dynamic, and after 20 seconds a zebra pattern with moderate polarization appeared (Fig. 3), as well as two zebra stripes around $3 \mathrm{GHz}$ at 05:00:56 UT that became more pronounced in right polarization, and at this time they consisted of spikes. The Nobeyama data show at this moment a double radio source above the neutral magnetic line, and the main, stronger radio emission (L-sign) corresponds to the ordinary mode. An enlarged fragment (3.54 s) of zebra stripes at the beginning of this interval is shown at the bottom of Fig. 3. We can see that the majority of spikes have a duration of one pixel, i.e. $\leq 8 \mathrm{~ms}$.

The bandwidth of spikes is equal to about $35-45 \mathrm{MHz}$, and $\Delta f_{\mathrm{e}} \approx 60 \mathrm{MHz}$. Spikes in two neighboring zebra-stripes do not have any correlation, as shown in Fig. 4 with a multichannel presentation of two short fragments of zebra patterns from the Fig. 3: between 05:00:45, 5-05:00:48,6 UT in left polarization and between 05:00:52, 5-05:00:57,0 UT in right polarization. The frequencies of channels were chosen almost along 

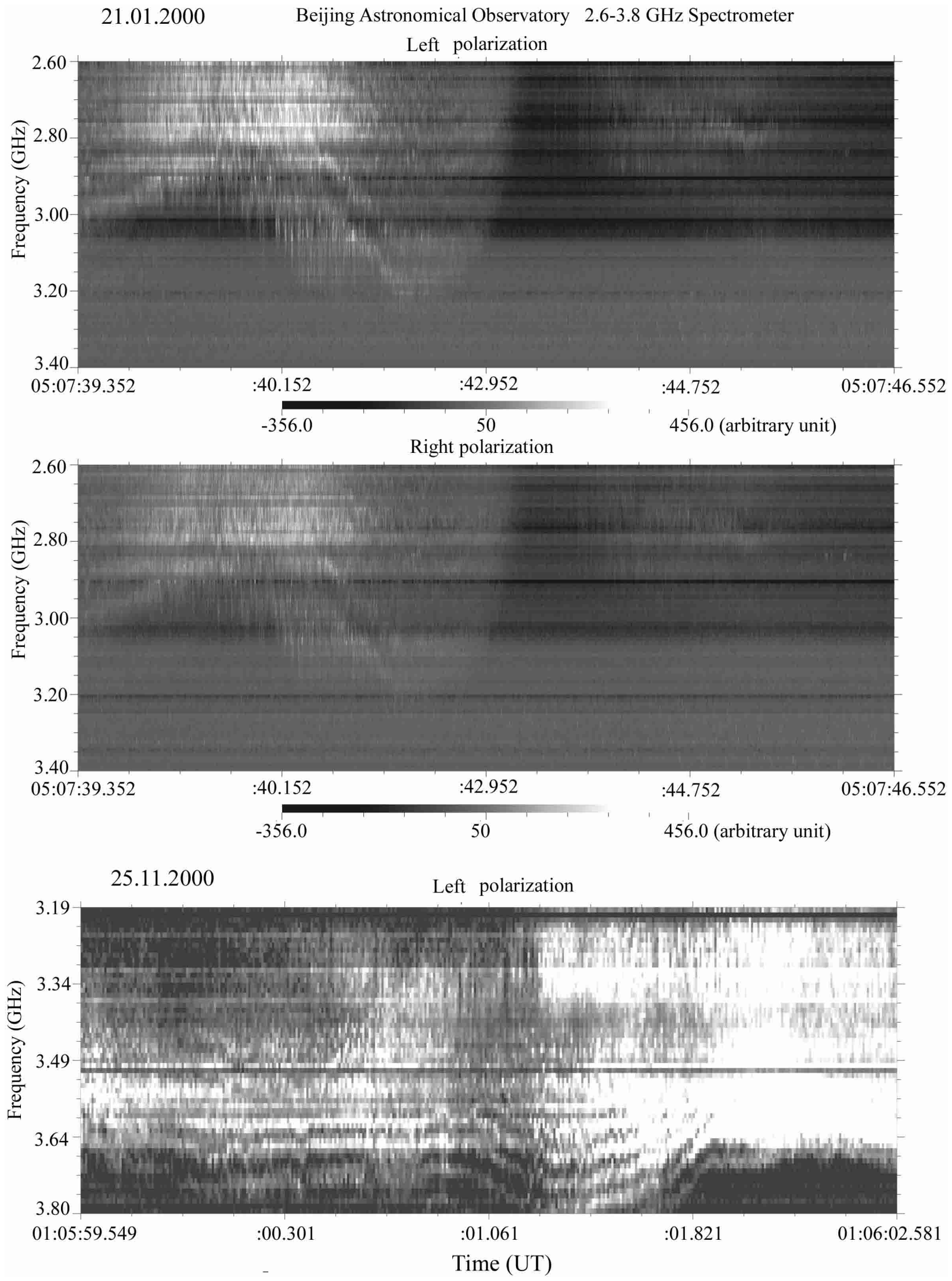

Fig. 1. Dynamical spectra of the superfine structure of a zebra pattern in two events: 21 January 2001 (two top panels in left and right circular polarization in the frequency range 2.6-3.4 GHz) and 25 November 2000 (bottom panel of $\sim 3 \mathrm{~s}$ duration in left polarization in the range 3.19-3.8 GHz). 


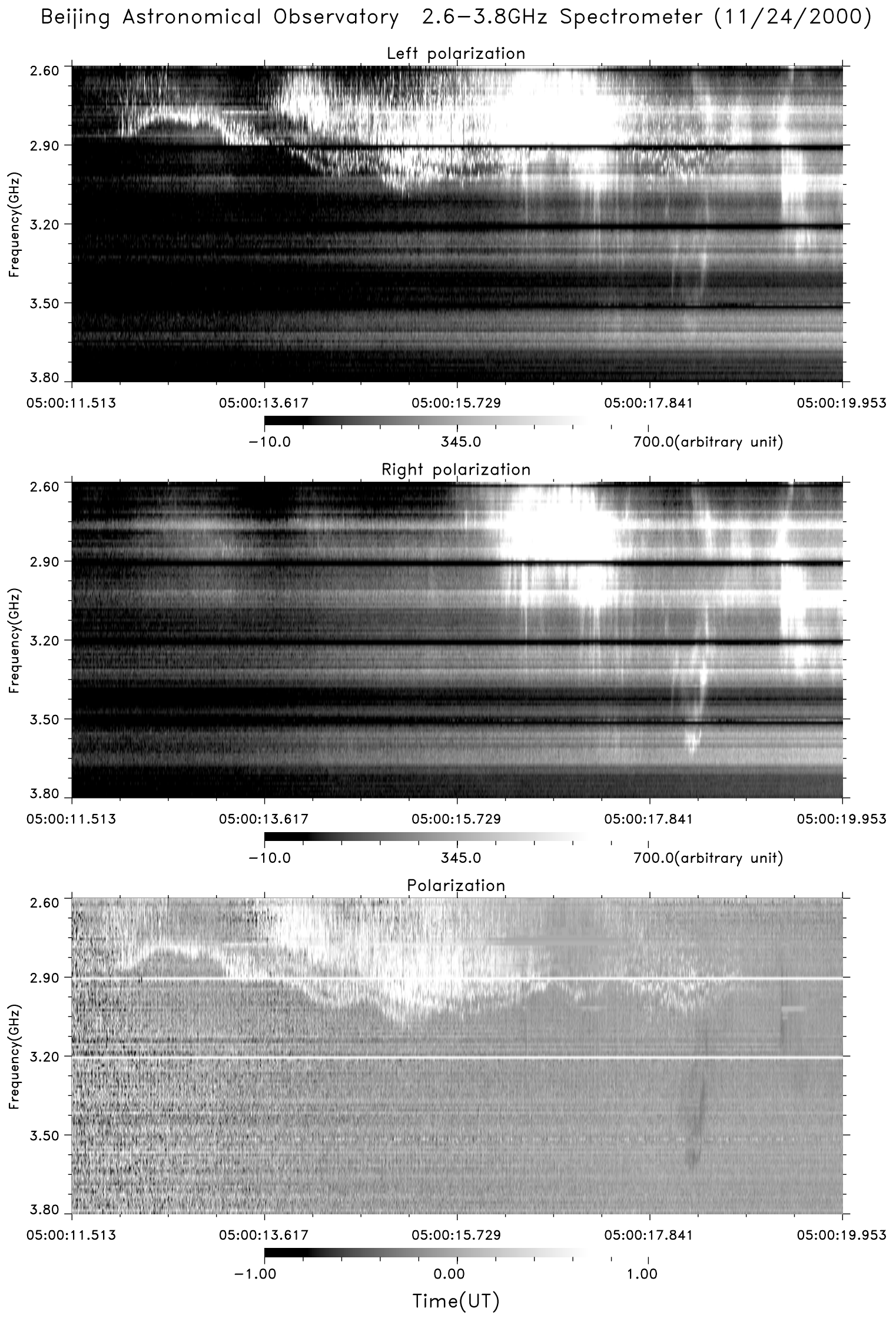

Fig. 2. The event of 24 November 2000 in the range 2.6-3.8 GHz: an isolated zebra stripe, consisting of millisecond spikes, evolves over $6 \mathrm{~s}$ into double and triple stripes. 


\section{Beijing Astronomical Observatory 2.6-3.8GHz Spectrometer (11/24/2000)}
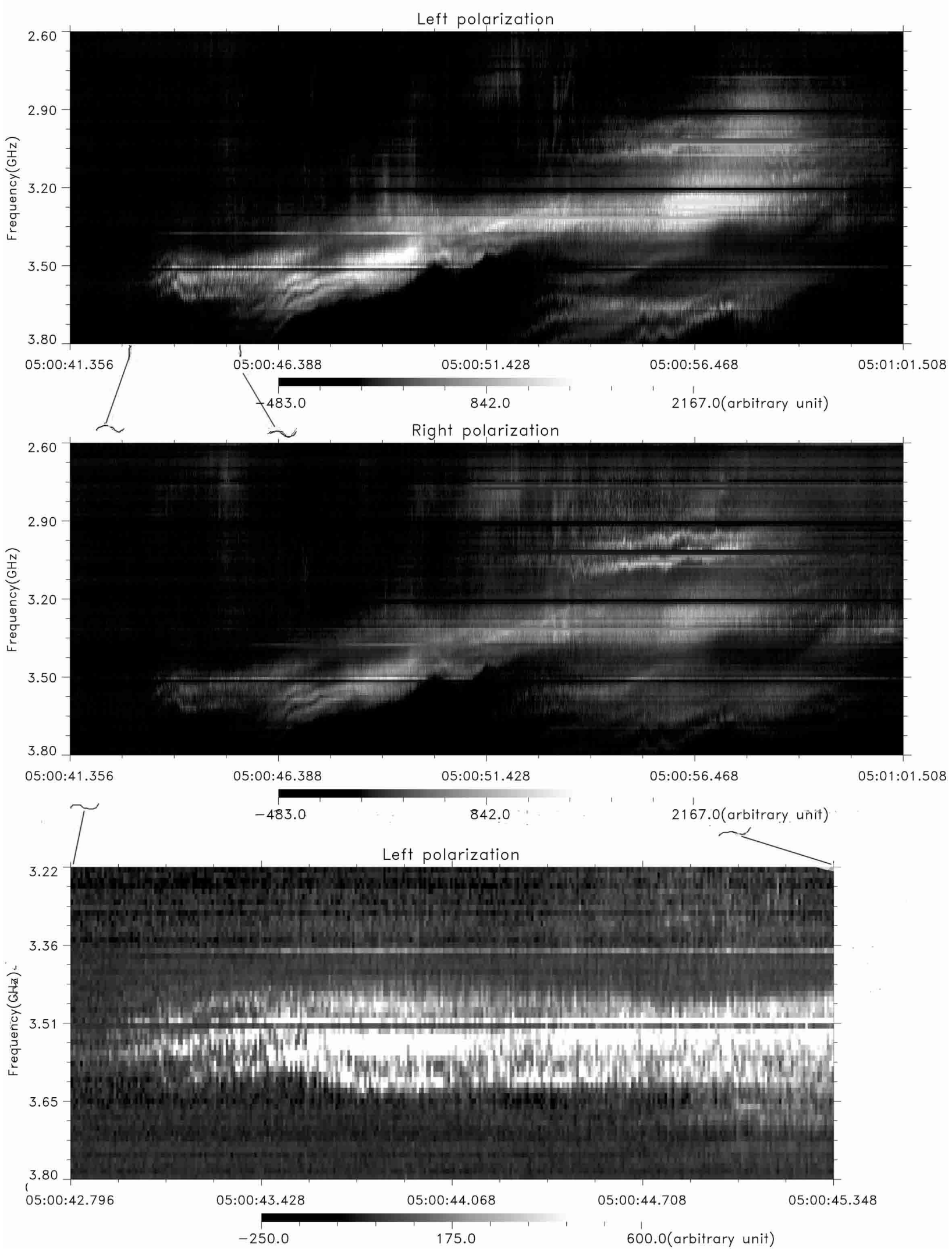

Fig. 3. The continuation of the event of 24 November 2000 showing a complex zebra-pattern with superfine structure. An enlarged fragment (3.54 s) of zebra-stripes in left polarization at the beginning of this interval is shown on the bottom panel. The radio emission on the whole has a moderate left polarization, but two zebra stripes around $3 \mathrm{GHz}$ and 05:00:56 UT have a moderate right polarization. 

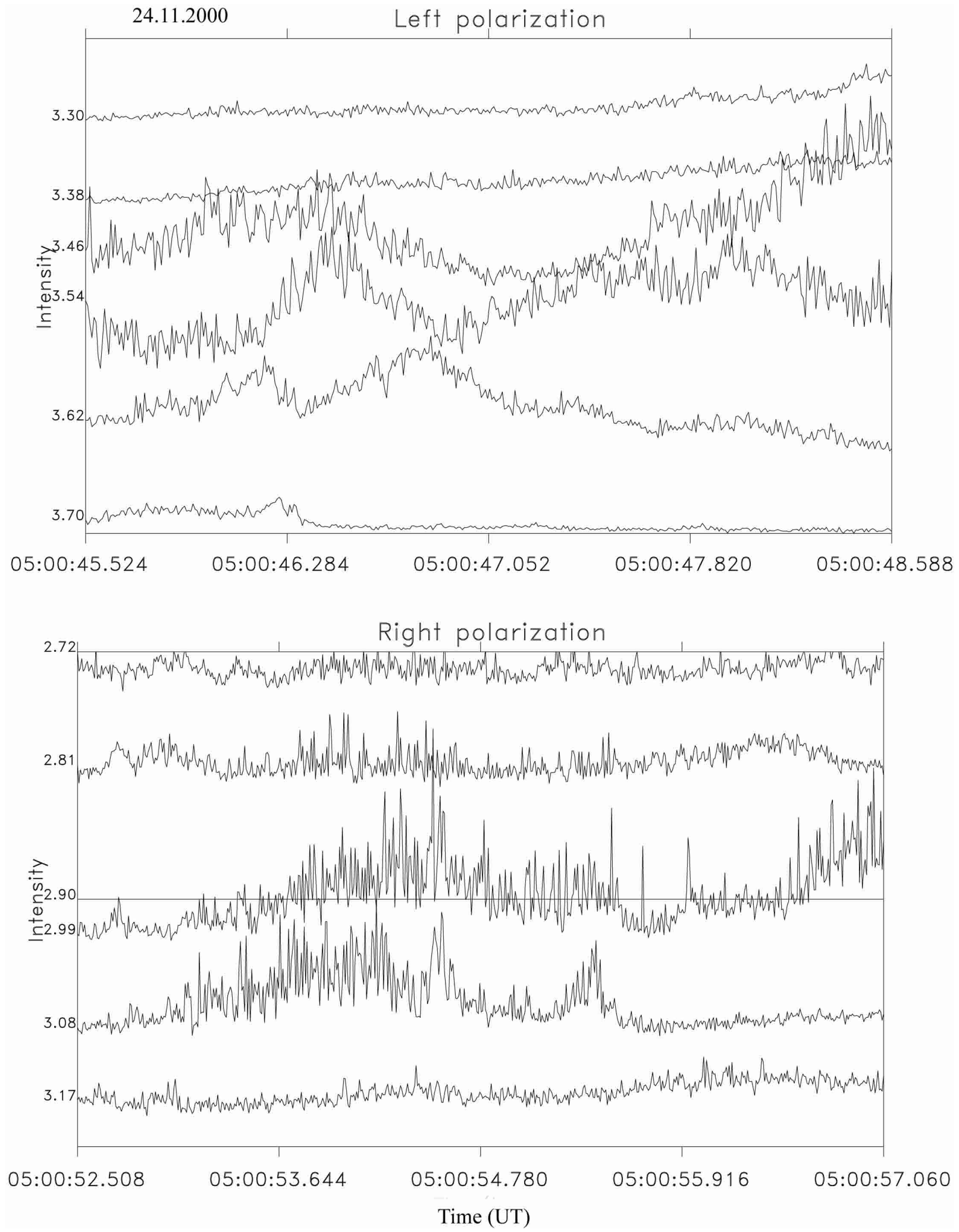

Fig. 4. A multichannel presentation of two fragments of ZPS from Fig. 3 with a duration of $3 \mathrm{~s}$ (in left polarization) and $4,5 \mathrm{~s}$ (in right polarization). The frequencies of channels were chosen almost along zebra-stripes in emission.

zebra-stripes in emission. We see obvious chaotic behaviour of spikes, as in practically all such events. Multichannel intensity profiles show also a very important result: the intensity between zebra stripes (in absorption stripes) remains lower than the intensity between spikes in emission stripes, but slightly higher than in parts of the spectrum without the background burst emission. Spikes almost disappear in absorption stripes, but the intensity level there testifies to a modulation effect. 

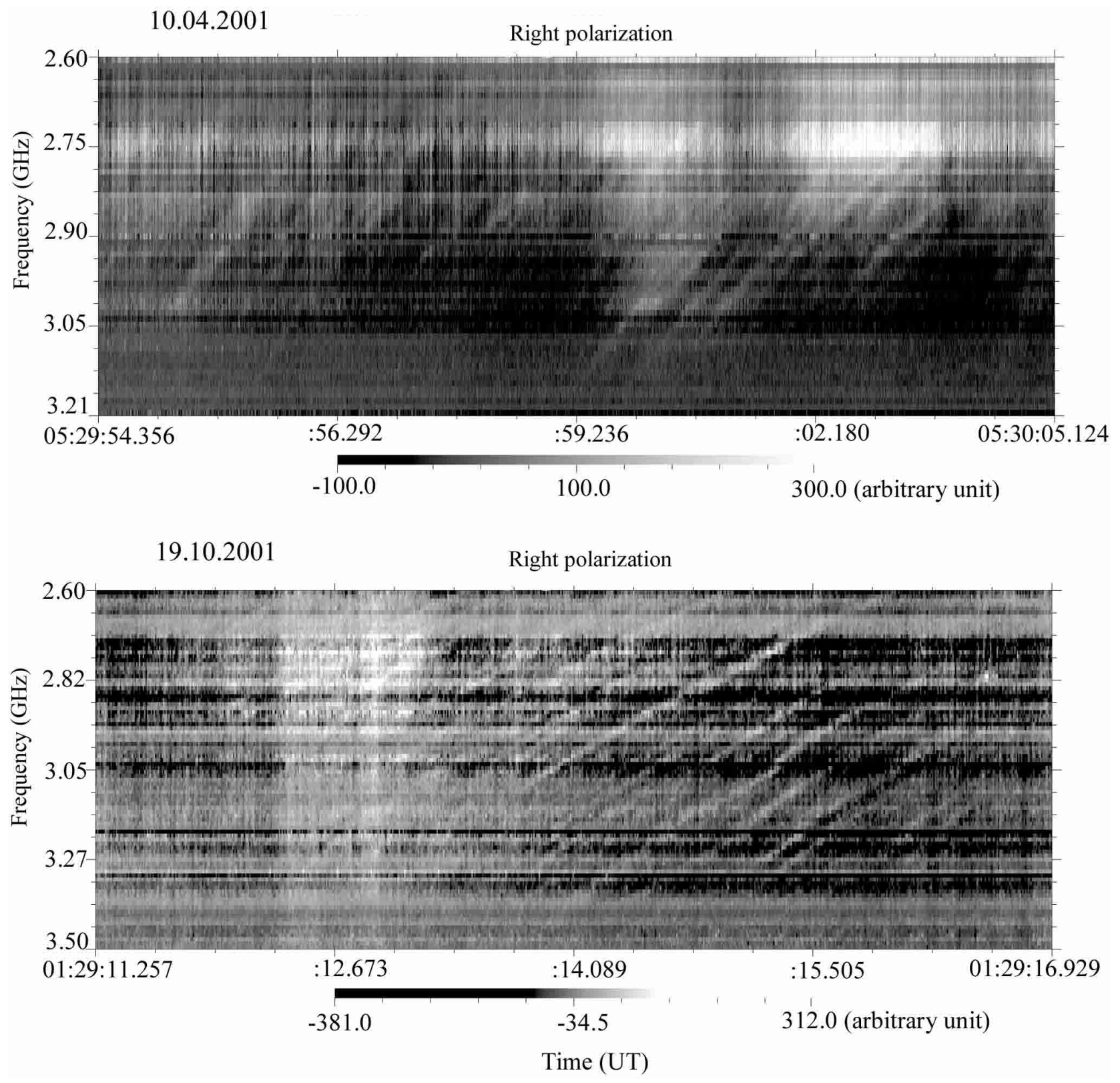

Fig. 5. Fiber-bursts superimposed on fast broadband pulsations in two events, 10 April and 19 October 2001. In both cases the emission was strong polarized. FB appeared without an obvious spiky structure, while ZPS appeared alternatively with FB and presented spiky superfine structure.

The flux density of spikes in the composition of zebra-stripes is usually about $20-50 \%$ (in different events) of the radiation of the background burst (their values are listed in the fifth column of the Table 1).

Another important observation was that the spiky structure is not pronounced in FB, when fibers appear simultaneously with ZPS (October 29, 2000, see Figs. 2 and 4 in Chernov et al. 2001), or alternately with ZPS as shown in Fig. 5 with two fragments of FB in the events of April 10, 2001 and October 19, 2001. In both these fragments, FB are superimposed on broadband fast pulsations. Such a superimposition is a wellknown effect in meter and decimeter ranges. Zebra stripes as well as fibers can appear as isolated stripes, and all other parameters of both stripes in emission and in absorption are similar (Chernov et al. 2001b). We conclude that the spiky structure of ZPS is seen almost in all events, when the time resolution of the spectrometer is high enough: some ms. In this connection the rapid frequency variations in the range $0.8-1.3 \mathrm{GHz}$ observed by Karlicky et al. (2001) with $0.1 \mathrm{~s}$ resolution (called Lace-bursts) are possibly similar structures if observed with millisecond resolution.

\section{Discussion}

\subsection{Emission mechanism}

\subsubsection{Double plasma resonance}

According to Table 1, the number of stripes in ZPS is often as high as 6. For example, in the bottom of Fig. 1, in left polarization with a well pronounced zebra pattern one sees about 6 
zebra bands between 3.5 and $3.8 \mathrm{GHz}$, so the separation is $\Delta f_{\mathrm{e}}=0.3 \mathrm{GHz} / 5=60 \mathrm{MHz}$. If one interprets the zebra bands as harmonics of the gyrofrequency, $f_{\mathrm{UH}}=s \times f_{\mathrm{Be}}$ one would get a gyrofrequency of $f_{\mathrm{Be}}=60 \mathrm{MHz}$, which corresponds to $B \approx 20 \mathrm{G}$, and a harmonic of $s=3600 / 60=60$. If we believe the model at the double plasma resonance of Zheleznyakov \& Zlotnik (1975) or Winglee \& Dulk (1986), $s>20$ are unlikely at the fundamental frequency $f_{\mathrm{UH}}$. We cannot believe the emission at the second harmonic of $f_{\mathrm{UH}}$, because in most cases the zebra pattern is strongly polarized. But at the frequency near $3.5 \mathrm{GHz}$ a density of $n_{\mathrm{e}}=1.5 \times 10^{11} \mathrm{~cm}^{-3}$ and a magnetic field of $B=20 \mathrm{G}$ is not unreasonable near a reconnection site in a flare loop. At the same time an interpretation in terms of the sum of the local upper hybrid frequency and low harmonics of the electron frequency $f=f_{\mathrm{UH}}+s f_{\mathrm{Be}}$ is less likely, because it requires multi-step processes of wave coupling from a compact radio source.

In two recent papers (Karlicky et al. 2001; Barta \& Karlicky 2001) it is supposed that the radio emission at double plasma resonance is generated in plasma with rapidly changing plasma parameters in turbulent magnetic loops. A possible source of the turbulence can be the plasma outflows from the magnetic field reconnection. It is supposed that scales of heights of density and the magnetic field in a loop vary chaotically along the loop, and the condition of double plasma resonance can be realized only at certain points along a loop. It is supposed that the area around each such point can be a source of spike emission accompanying ZPS. However, the authors do not discuss processes that could result in such a loop structure with strongly turbulent plasma. Thus spikes can form chaotic chains, however the occasional realization of a double plasma resonance (both in one loop and in neighboring ones) excludes formation of parallel drifting zebra-stripes.

Recent SOHO and TRACE data suggest narrow pre-flare loops, along which the density and magnetic field change very slowly (Aschwanden et al. 1999, 2002), (when density and magnetic field scale heights are about the same, $\sim 55 \pm 10 \mathrm{Mm}$, comparable with the length of the loop). This proves that double plasma resonance surfaces cannot exist in such loops.

In Karlicky et al. (2001) and Barta \& Karlicky (2001) the nonlinear process that causes saturation of the instability is not studied in detail, nor are the wave transformation processes. Notice that in our case spikes as superfine structure of zebra stripes have much smaller duration $(8 \mathrm{~ms})$. For an explanation of the discussed structure we will consider alternative models of whistlers and ion-sound waves, in which the radio emission is formed as a result of the more effective interactions: $l+s \rightarrow t$ and $l+w \rightarrow t$.

\subsubsection{Is- and /w- interactions}

ZPS and FB have almost the same main parameters (except the frequency drift), therefore both structures may have similar natures. Similar to the situation in meter and decimeter ranges (Chernov 1976; Chernov et al. 1998), we propose as such a mechanism the coupling of electrostatic plasma waves with whistlers, in different conditions of whistler instability.
We explain the multiple, equi-spaced zebra bands by the whistler spectrum with periodic wave packets along the magnetic loop, due to the periodic whistler cyclotron instability, the scattering on the ambient plasma and to the bounce motion of fast particles in the magnetic trap. Even a narrow magnetic loop should consist of narrow zones of strong whistler instability, shared periodically by decay zones, and a system of multiple zebra-lines is radiated from the distributed source along the magnetic trap (Maltseva \& Chernov 1989). The conservation laws also apply for the interaction of plasma waves on the upper hybrid frequency with whistlers, propagating along large angles to the magnetic field (excited with anomalous Doppler resonance).

Microwave spikes are usually observed more often than ZPS and FB, even without these structures, and we must ascertain why ZPS at microwaves appear mainly with a superfine spiky structure and FB practically without any such structure.

Microwave spikes as a part of ZPS have millisecond durations, and for such spikes a new model was proposed in Chernov et al. (2001a), namely the coupling of plasma and ion-sound waves: $l+s \rightarrow t$ in a radio source, related to fast shock fronts in the flare region. The behaviour of ion-sound waves and whistlers in coronal magnetic traps was considered in Chernov (1989), where it was shown that a dynamic energy transfer between $(s)$ - waves and whistlers can operate in a pulsating regime with the processes $s+s^{\prime} \rightarrow w$. The efficiency of these processes becomes maximal when the whistler frequency is about equal to the ion plasma frequency: $\omega_{w} \sim \omega_{\mathrm{Pi}}$. In the meter and decimeter ranges such a coincidence takes place when $\omega_{w} \sim(0.1-0.25) \omega_{\mathrm{Be}}$ (see Fig. 1 in Chernov 1989). In our case the generation of whistlers occurs in the flare region, where the plasma temperature is higher than in the corona. According Yohkoh/SXT data it may be $\geq 10^{7} \mathrm{~K}$ (Chernov et al. 2001a), and whistler instability displaces to lower frequencies (Berney \& Benz 1978), and according to estimations in Chernov et al. (2001b), $\omega_{w} \leq 0.1 \omega_{\mathrm{Be}}$. In such a case the condition $\omega_{w} \sim \omega_{\mathrm{Pi}}$ should be realized in the microwave range around $3 \mathrm{GHz}$.

\subsection{The model of radio source}

All new optical and X-ray data show that a chromospheric flare is caused by the magnetic reconnection of a new emerging magnetic loop with a preexisting one (Aschwanden 2002). In the general case, the current sheet should be inclined, and arises due to the approach of both loops, as is shown in Fig. 6 (similar to examples in Fig. 6.5b in Priest 1982 and in Yokoyama \& Shibata 1996). In such a case the fast shock wave propagates upwards from the X-point of magnetic reconnection, and instabilities of high frequency plasma waves and low frequency waves (ion-sound waves and whistlers) develop in the shock front. Moreover the best conditions are fulfilled $\left(\omega_{w} \sim \omega_{\mathrm{Pi}}\right)$ for the pulsating regime of the whistler spectrum due to the process $s+s^{\prime} \rightarrow w$. Thus, the radio emission could be modulated by fast spikes as a result of the process: $l+s \rightarrow t$, (see details in Chernov 1989) and simultaneously by ZPS and FB due to 


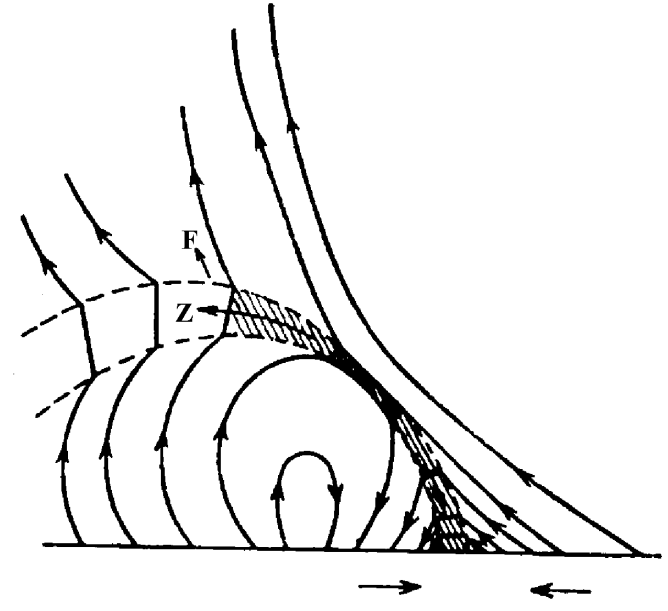

Fig. 6. A scheme of the probable relation between radio sources of FB and ZPS with an inclined current sheet in the reconnection region with approaching magnetic loops.

the process $l+w \rightarrow t$. In both processes radio emission escapes in O-magnetoionic mode. If the velocity of plasma flow from a current sheet is not high enough to form the fast shock front, then s-waves can be generated in the current sheet, and with a low efficiency of whistler instability (without the loss-cone distribution of fast particles), only millisecond spikes (and fast pulsations) can be generated, which we observe more often (Chernov 1989).

Upstream of the fast shock front, whistlers should be generated by fast particles with loss-cone distribution. Whistlers generated at the normal Doppler cyclotron resonance can escape along the main magnetic loop and yield FB (a probable position of FB radio source is shown by $F$ in Fig. 6). Whistlers with the anomalous Doppler effect are generated under large angles to magnetic force lines and they form standing wave packets in front of the shock wave (shown by $\mathrm{Z}$ in Fig. 6). This is a case when the group velocity of whistlers is about equal to the velocity of the shock, that yields ZPS with slow oscillating frequency drift (Chernov 1989; Maltseva \& Chernov 1989).

The process $s+s^{\prime} \rightarrow w$ has a limitation with regard to the angles of whistler wave vectors to the magnetic force line (Chernov 1989) (they must be $>70^{\circ}$ ), that explains why the spiky structure is almost not present in FB: because in this case whistlers propagate along magnetic loops. The shock wave in the downward direction is more rare, because the Alfven velocity is higher in regions with higher magnetic field strength. Therefore we propose that in all our events the radio source was located upstream of an upward shock, otherwise a structure of narrowband rope-like fibers should be generated, as was shown in Chernov (1990) (and in detail in Chernov 1997). We did not observe such rope-like FB or slowdrifting fibers in absorption, that are also characteristic of a radio source localized in a rarefaction region between two fast shocks and four slow shocks surrounding the $\mathrm{X}$-point of the magnetic reconnection (Chernov 1997).

\subsection{Wave-wave interactions and radio emission}

From Chernov et al. (2001a) it is clear that the $l s$-interaction is very similar to the interaction of plasma waves with whistlers (Fomichev \& Fainstein 1988; Chernov \& Fomichev 1989) However, in the last process whistler wave numbers are considerably limited (from below and from above). For the rest, these two interactions are equally efficient.

According to Tsytovich (1971) and Melrose (1980, 1983) the interaction of high- and low-frequency waves can take place either at the sum frequency or at the difference frequency. Coalescence and decay can be equally efficient processes. The kinetic equations for the rate of growth of transverse waves in the processes $l+w \rightarrow t$ and $l \rightarrow t+w$ differ only by the signs before the second and third terms in the equation (Eq. (B.2) in Melrose 1980):

$\frac{\mathrm{d} N_{ \pm}^{t}}{\mathrm{~d} t}=\int \frac{\mathrm{d}^{3} \boldsymbol{k}^{l}}{(2 \pi)^{3}} \int \frac{\mathrm{d}^{3} \boldsymbol{k}^{w}}{(2 \pi)^{3}} u_{ \pm}^{t l w}\left[N^{l} N^{w} \mp N^{t}\left(N^{l} \pm N^{w}\right)\right]$

(the upper signs for coalescence and the lower signs for decay), where $N^{t, l, w}$ are dimensionless wave intensities. The only difference in the equations for the coalescence and decay probabilities $u_{ \pm}^{t l w}$ is in the signs of the resonance conditions in the argument of the delta function.

By analogy to Fomichev \& Fainstein (1988) and with allowance for the abovementioned sign changes in the kinetic equation, for given intensities $N^{l, w}$ the following equation holds for $l w$-interactions:

$\mathrm{d} N^{t} / \mathrm{d} z=\Gamma_{1} \mp N^{t} \Gamma_{2}$,

where $\Gamma_{1}=\frac{1}{4}\left(\frac{\omega_{w}}{\omega_{\mathrm{Be}}}\right)^{3 / 4}\left(\frac{\omega_{\mathrm{Be}}}{\omega_{\mathrm{P}}}\right)^{2} N_{l} N_{w}$;

$\Gamma_{2}=\frac{1}{4}\left(\frac{\omega_{w}}{\omega_{\mathrm{Be}}}\right)^{1 / 4}\left(\frac{\omega_{\mathrm{Be}}}{\omega_{\mathrm{P}}}\right)^{3} N_{l}-\frac{1}{4}\left(\frac{\omega_{w}}{\omega_{\mathrm{Be}}}\right)^{3 / 4} \frac{\omega_{\mathrm{Be}}}{\omega_{\mathrm{P}}} N_{w} ;$

and $z=\frac{\omega_{\mathrm{P}}}{c} v_{g r}^{t} t \frac{\omega_{\mathrm{P}}}{\Delta \omega_{w}}$ is the dimensionless length along the magnetic field. The solution of this linear nonhomogeneous equation with start condition $N_{t}(0)=0$ is:

$N_{t}(z)=\Gamma_{1} / \Gamma_{2}\left(1-\exp ^{-\Gamma_{2} z}\right)$

It is known that both processes ( $l s-$ and $l w$-interactions) become efficient only in an optically thick source (Benz \& Wentzel 1981; Fomichev \& Fainstein 1988).

According to Benz \& Wentzel (1981) $l s$-interaction becomes most efficient with a high intensity of $s$ waves when the optical thickness unity occurs over only a few meters.

It is easily to show that in an optically thick source the interactions at the frequencies $\omega_{t}=\omega_{l} \pm \omega_{s}$ can be equally efficient and the maximum of brightness temperature can be derived (similar to the procedure in Benz \& Wentzel 1981 and in Chernov \& Fomichev 1989):

$T_{\mathrm{b}}=\frac{h \omega_{\mathrm{Pe}}}{k_{\mathrm{B}}} \frac{N_{s} N_{l}}{N_{s} \pm N_{l}}$,

where $h$ is Plank's constant, $k_{\mathrm{B}}$ is Bolzmann's constant and $N_{l}$, $N_{s}$ are dimensionless wave intensities $\left(N_{l} \ll N_{s}\right)$ (or wave quantum densities). That is the theoretical estimation. From observation we obtain

$T_{\mathrm{b}}=\frac{2 \pi^{2}}{k_{\mathrm{B}}} \frac{W_{t}}{k_{t}^{2} \Delta k_{t}}=\frac{0.143 \times 10^{7} S(\mathrm{sfu})}{f^{2} l_{10}^{2}} \exp ^{\tau}$, 
where the designations are similar to Fomichev \& Fainstein (1988): $\tau$ is the optical depth of the solar corona for the electromagnetic emission, $S$ (sfu) - the burst flux in solar flux units, $f$-frequency in $\mathrm{GHz}$ and $l_{10}$ - linear source size perpendicular the line of sight in units of $10^{10} \mathrm{~cm}$. So that with the moderate flux emission $100 \mathrm{sfu}$ and burst source $10^{8} \mathrm{~cm}$ we obtain the observed $T_{\mathrm{b}} \approx 7 \times 10^{13} \mathrm{~K}$, i.e. close to the value predicted in Benz $\&$ Wentzel (1981), for instance with a real small value of density energy of plasma wave $W_{l} \approx 10^{-7} \mathrm{erg} / \mathrm{cm}^{3}$ for isotropic $l$-waves, and $W_{l}=\frac{h}{(2 \pi)^{3}} \omega_{\mathrm{Pe}} N_{l} k_{l}^{2} \Delta k_{l} \Omega_{l}$. So with a solid angle $\Omega_{l} \leq 1, k_{l} \approx \Delta k_{l} \approx 5 \times 10^{-2} \mathrm{~cm}^{-1}$ we receive from (6) for $N_{l} \ll N_{s} T_{\mathrm{b}} \approx 5,7 \times 10^{13} \mathrm{~K}$. Thus, the discussed emission processes ( $l s$ - and $l w$-interactions) provide the necessary brightness temperatures.

\section{Conclusion}

We have described the new effect of superfine structure in microwave radio bursts: zebra patterns consisting of millisecond spikes. We conclude that the spiky structure of ZPS is observed practically in all events, when the time resolution of the spectrometer is high enough: some ms. Both cyclotron and plasma emission of nonthermal electrons generally contributes to microwave burst emission. The absence of any correlation between spikes in two neighboring zebra stripes testifies to different spacial locations of radio sources of neighboring zebrastripes, for small values of spike source dimension. However, according to the latest SOHO and TRACE data testifying to narrow flare loops, along which the density and magnetic field vary very slowly (Aschwanden et al. 1999), the mechanism of double plasma resonance cannot be realized in such loops.

For the observed superfine structure, the coupling of plasma electrostatic waves with whistlers is the most plausible, taking into account the pulsating regime of the coupling process of whistlers with ion-sound waves. The zebra-pattern consisting of spikes can be identified as simultaneous manifestations of the processes $(l+s \rightarrow t$ and $l+w \rightarrow t)$ in radio sources, related to magnetic reconnections above flare regions. Such a scheme explains their main spectral properties, as well as the polarization in O-mode. Changes of polarization are usually related to the double structure of radio sources above different magnetic polarities.

Acknowledgements. G.P.Ch. is grateful for the support of the Chinese Academy of Sciences and NSF of China that enabled him to work with colleagues at NAOC, and the Russian Foundation of Basic Research, grant No. 02-02-16201. The Chinese authors acknowledge support of NSFC and Ministry of Sciences and Technology of China.

\section{References}

Altyntsev, A. T., Grechnev, V. V., \& Hanaoka, Y. 1998, Sol. Phys., 178,575

Aschwanden, M. J. 2002, Particle acceleration and kinematics in solar flares (Dordrecht: Kluwer Ac. Publ.)

Aschwanden, M. J., Newmark, J. S., Delabouniere, J.-P., et al. 1999, ApJ, 515, 842

Barta, M., \& Karlicky, M. 2001, A\&A, 379, 1045

Benz, A. O. 1993, Plasma Astrophysics, Kinetic processes in solar and stellar Coronae (Dordrecht: Kluwer Academic Publisher)

Benz, A. O., \& Wentzel, D. 1981, A\&A, 94, 100

Berney, M., \& Benz, A. O. 1978, A\&A, 65, 369

Chernov, G. P. 1976, Soviet Astron., 20, 582

Chernov, G. P. 1989, Soviet Astron., 33, 649

Chernov, G. P. 1990, Sol. Phys., 130, 75

Chernov, G. P. 1996, Astron. Rep., 40, 561

Chernov, G. P. 1997, Astron. Lett., 23, 827

Chernov, G. P., Markeev, A. K., Poquerusse, M., et al. 1998, A\&A, 334,314

Chernov, G. P., Fu, Q. J., Lao, D. B., \& Hanaoka, Y. 2001a, Sol. Phys., 201, 153

Chernov, G. P., Yasnov, L. V., Yan, Y. H., \& Fu, Q. J. 2001b, Chin. J. Astron. Astrophys., 1, 525

Fleishman, G. D., \& Melnikov, V. F. 1998, Physics Uspekhi, 168, 1265

Fu, Q. J., Qin, J. H., Ji, H. R., \& Pei, L. B. 1995, Sol. Phys., 160, 97

Isliker, H., \& Benz, A. O. 1994, A\&AS, 104, 145

Karlicky, M., Barta, M., Jiricka, K., Meszarosova, H., Fernandes, F. C. R., \& Cecatto, J. R. 2001, A\&A, 375, 638

Kuijpers, J. 1975, Sol. Phys., 44, 173

Kuijpers, J., Van der Post, P., \& Slottje, C. 1981, A\&A, 102, 331

Ledenev, V. G., Karlicky, M., Yan, Y., \& Fu, Q. 2001, Sol. Phys., 202, 71

Maltseva, O. A., \& Chernov, G. P. 1989, Kinematika i Fizika Nebesnykh Tel, 5, 39

Melrose, D. B. 1980, Sol. Phys., 67, 357

Melrose, D. B. 1983, Sol. Phys., 87, 359

Mollwo, L. 1983, Sol. Phys., 83, 305

Mollwo, L. 1988, Sol. Phys., 116, 323

Priest, E. R. 1982, Solar Magnetohydrodynamics (Dordrecht: D. Reidel Publishing Company)

Sawant, H. S., Karlicky, M., Fernandes, F. C. R., \& Cecattto, J. R. 2002, A\&A, 396, 1015

Slottje, C. 1981, Atlas of fine structures of dynamic spectra of solar type IV-dm and some type II bursts (Utrecht Observatory)

Tsytovich, V. N. 1971, Nonlinear Effects in Plasma (New York: Plenum)

Winglee, R. M., \& Dulk, G. A. 1986, ApJ, 307, 808

Yokoyama, T., \& Shibata, K. 1996, Publ. Astron. Soc. Japan, 48, 353

Zheleznyakov, V. V., \& Zlotnik, E. Ya. 1975, Sol. Phys., 44, 461 